\title{
Infective endocarditis caused by Salmonella enteritidis in a dialysis patient: a case report and literature review Yusuke Tsugawa*†1, Miyuki Futatsuyama ${ }^{\dagger 1}$, Keiichi Furukawa ${ }^{\dagger 2}$, Fumika Taki $^{\dagger 1}$, Yuji Nishizaki ${ }^{\dagger 1}$, Keiichi Tamagaki ${ }^{\dagger 1}$, Yuki Kaneshiro ${ }^{\dagger 1}$ and Yasuhiro Komatsu ${ }^{\dagger 1}$
}

Address: ${ }^{1}$ Department of Nephrology, Division of Internal Medicine, St. Luke's International Hospital, Tokyo, Japan and ${ }^{2}$ Department of Infectious Disease, Division of Internal Medicine, St. Luke's International Hospital, Tokyo, Japan

Email: Yusuke Tsugawa* - yusuke@luke.or.jp; Miyuki Futatsuyama - nakamiyu@luke.or.jp; Keiichi Furukawa - keifuru@luke.or.jp; FumikaTaki - ftaki@luke.or.jp; Yuji Nishizaki - nishiyu@luke.or.jp; Keiichi Tamagaki - keitama@luke.or.jp; Yuki Kaneshiro - kaneyuki@luke.or.jp; Yasuhiro Komatsu - komayasu@luke.or.jp

* Corresponding author †Equal contributors

Published: 29 September 2009

BMC Infectious Diseases 2009, 9:161 doi:10.1186/|47|-2334-9-161
Received: II June 2009

Accepted: 29 September 2009

This article is available from: http://www.biomedcentral.com//47/-2334/9//6I

(c) 2009 Tsugawa et al; licensee BioMed Central Ltd.

This is an Open Access article distributed under the terms of the Creative Commons Attribution License (http://creativecommons.org/licenses/by/2.0), which permits unrestricted use, distribution, and reproduction in any medium, provided the original work is properly cited.

\begin{abstract}
Background: Infective endocarditis is significantly more common in haemodialysis patients as compared with the general population, the causative pathogen is generally Staphylococcus aureus; there have been no previously reported cases of infective endocarditis caused by a Salmonella species in haemodialysis patients.

Case Presentation: We report the case of a 68 year-old woman on haemodialysis who developed infective endocarditis as a result of Salmonella enteritidis. Although we treated the patient with ceftriaxone combined with ciprofloxacin, infective endocarditis was not detected early enough and unfortunately developed into cerebral septic emboli, which ultimately resulted in death.

Conclusion: Although there are several reports that Salmonella endocarditis without cardiac failure can be successfully treated with antibiotics alone, early surgical intervention is essential for some cases to prevent life-threatening complications. Transesophageal echocardiography should be performed in any patient with high clinical suspicion of infective endocarditis. To the best of our knowledge, this is the first case-report of Salmonella endocarditis in a haemodialysis patient.
\end{abstract}

\section{Background}

Infective endocarditis is significantly more common in haemodialysis patients as compared with the general population. In addition to the four conventional categories of infective endocarditis (native valve infective endocarditis, prosthetic valve infective endocarditis, infective endocar- ditis in intravenous drug users, and nosocomial infective endocarditis), a fifth category, health-care associated infective endocarditis or haemodialysis associated infective endocarditis, has recently been proposed. Although infective endocarditis is a common complication among haemodialysis patients, the causative pathogen is gener- 
ally Staphylococcus aureus; there have been no reported cases of infective endocarditis caused by a Salmonella species in haemodialysis patients. Salmonella species have been reported to cause bacteremia [1] and infective endocarditis in 5 and $1.4 \%$ of patients [2] during gastrointestinal infections, respectively. However, in general, cases of infective endocarditis due to Salmonella species are relatively rare. Here, we report the case of a 68 year-old woman on haemodialysis who developed infective endocarditis on her native mitral valve as a result of Salmonella enteritidis. After hospitalization, the patient was treated with intensive antibiotic therapy. However, the infective endocarditis was not detected early enough and unfortunately developed into cerebral septic emboli, which ultimately resulted in death.

\section{Case Presentation}

A 68 year-old Asian woman was admitted to our hospital presenting with a two-day history of high grade fever, diarrhea and nausea. The patient's symptoms began one day after eating grilled chicken and beef at a restaurant; however, she did not complain of abdominal pain, vomiting, or hematochezia. The patient was diagnosed with diabetes mellitus at the age of 50, had undergone haemodialysis due to diabetic nephropathy for the past 2 years, and was under three times weekly haemodialysis. She had also undergone an aortic mechanical valve replacement operation for aortic stenosis 7 months prior to the current admission.

Upon admission to our hospital, the patient's height was $149 \mathrm{~cm}$ and her body weight were $61.0 \mathrm{~kg}$ (dry weight). The patient's temperature was $106^{\circ} \mathrm{F}$, blood pressure was $150 / 90 \mathrm{mmHg}$, heart rate was $152 / \mathrm{min}$, and respiratory rate was $24 / \mathrm{min}$. Cardiovascular examination revealed a Levine IV/VI systolic murmur at the right second intercostal space. Her bowel movements were slightly hypoactive and physical examination did not reveal any abdominal tenderness or rebound. No evidence of embolization including Osler node, Janeway lesion and petechiae was observed. No other abnormalities were noted on the systemic examination. Initial laboratory examinations revealed the following results: hemoglobin, $115 \mathrm{~g} / \mathrm{L}$ [110$146 \mathrm{~g} / \mathrm{L}]$; total leukocyte counts, 10300/ $\mu \mathrm{L}$ [3500-8200/ $\mu \mathrm{L}$ ] without left shift (Myelo 0.5\%, Meta 0.5\%, Stab 2.0\%, Seg $90.0 \%$, Lym 1.5\%, Mono 5.5\%); normal platelet counts, $1.42 \times 10^{3} / \mu \mathrm{L}\left[1.40-3.89 \times 10^{3} / \mu \mathrm{L}\right]$; significantly elevated C-reactive protein, $18.54 \mathrm{mg} / \mathrm{dL}[<0.30 \mathrm{mg} / \mathrm{dL}]$; $\mathrm{Na} 131 \mathrm{mEq} / \mathrm{L}$, K $4.3 \mathrm{mEq} / \mathrm{L}, \mathrm{Cl} 97 \mathrm{mEq} / \mathrm{L}$, HCO3- 18.9 $\mathrm{mmol} / \mathrm{L}$; BUN $43.6 \mathrm{mg} / \mathrm{dL}$, Cre $7.25 \mathrm{mg} / \mathrm{dL}$ (before haemodialysis); serum glucose, $173 \mathrm{mg} / \mathrm{dL}$; and HbA1c, 7.3\% [4.3-5.8\%]. In addition, the patient was tested negative for HIV antibodies.
Salmonella enteritis or campylobacter enteritis was suspected due to the history of eating grilled chicken. Treatment was initiated with ciprofloxacin $300 \mathrm{mg}$ q24hr, ceftriaxone $2 \mathrm{~g} \mathrm{q} 24 \mathrm{hr}$, and clindamycin $600 \mathrm{mg} \mathrm{q} 12 \mathrm{hr}$ intravenously, started on day of admission. Three days post-admission, a serial blood culture taken on admission revealed Salmonella enterica serotype Enteritidis (O9, Hg) that was sensitive to ceftriaxone ( $\mathrm{MIC}<=1$ ) and ciprofloxacin (MIC $<=0.25$ ) (Table 1). After detection of the causable microorganism, ceftriaxone and clindamycin treatment was ceased, and the patient was treated with ciprofloxacin alone. Since the patient's aortic valve was a prosthetic, and Salmonella species have a high affinity for intravascular intima, there was a concern about prosthetic infective endocarditis. Therefore, the patient underwent transthoracic echocardiography (TTE) on day 7; however, no abnormalities were detected by TTE. On day 9, the patient showed a sudden disturbance in mental status, left conjugate deviation, and right hemiplegia. Diffusionweighted imaging of the brain via MRI showed an acute cerebral infarction of the middle cerebral artery region (figure 1). Following this discovery, the TTE was re-examined, followed by a transesophageal echocardiography (TEE) on day 13. The TEE revealed floppy vegetation on the mitral valve, which was $12 \times 8 \mathrm{~mm}$ in size (figure 2 ). We could not find any aberrance of the replaced aortic valve. Although surgery to remove the vegetation was considered, acute cerebral infarction contraindicated her from undergoing the operation. The ciprofloxacin dose was increased to $400 \mathrm{mg}$ q $24 \mathrm{hr}$ and was combined with ceftriaxone $2 \mathrm{q} q 12 \mathrm{hr}$, on day 12 . Consequently, the patient was

Table I: Susceptibility of the Salmonella Enteritidis to the Antibiotics

\begin{tabular}{lcc}
\hline \multicolumn{1}{r}{ Antibiotics } & MIC & Sensitivity \\
\hline CEZ & $<=4$ & $\mathrm{R}$ \\
AMK & $<=2$ & $\mathrm{R}$ \\
TOB & $<=1$ & $\mathrm{R}$ \\
GM & $<=I$ & $\mathrm{R}$ \\
AZT & $<=I$ & $\mathrm{~S}$ \\
CTRX & $<=I$ & $\mathrm{~S}$ \\
CAZ & $<=I$ & $\mathrm{~S}$ \\
ABPC & $<=2$ & $\mathrm{~S}$ \\
PIPC & $<=4$ & $\mathrm{~S}$ \\
CPFX & $<=0.25$ & $\mathrm{~S}$ \\
ST & $<=20$ & $\mathrm{~S}$ \\
S/A & $<=2$ & $\mathrm{~S}$ \\
MEPM & $<=0.25$ & $\mathrm{~S}$ \\
CFPM & $<=I$ & $\mathrm{~S}$ \\
PIPC/TAZ & $<=4$ & $\mathrm{~S}$ \\
& & \\
\hline
\end{tabular}

Abbreviations: CEZ; cefazolin, AMK; amikacin, TOB; tobramycin, GM; gentamicin, AZT; aztronam, CTRX; ceftriaxone, CAZ; ceftazidime, ABPC; ampicillin, PIPC; piperacillin, CPFX; ciprofloxacin, ST; sulfamethoxazole-trimethoprim, S/A; ampicillin sulbactam, MEPM; meropenem, CFPM; cefepime, PIPC/TAZ; piperacillin-tazobactam 


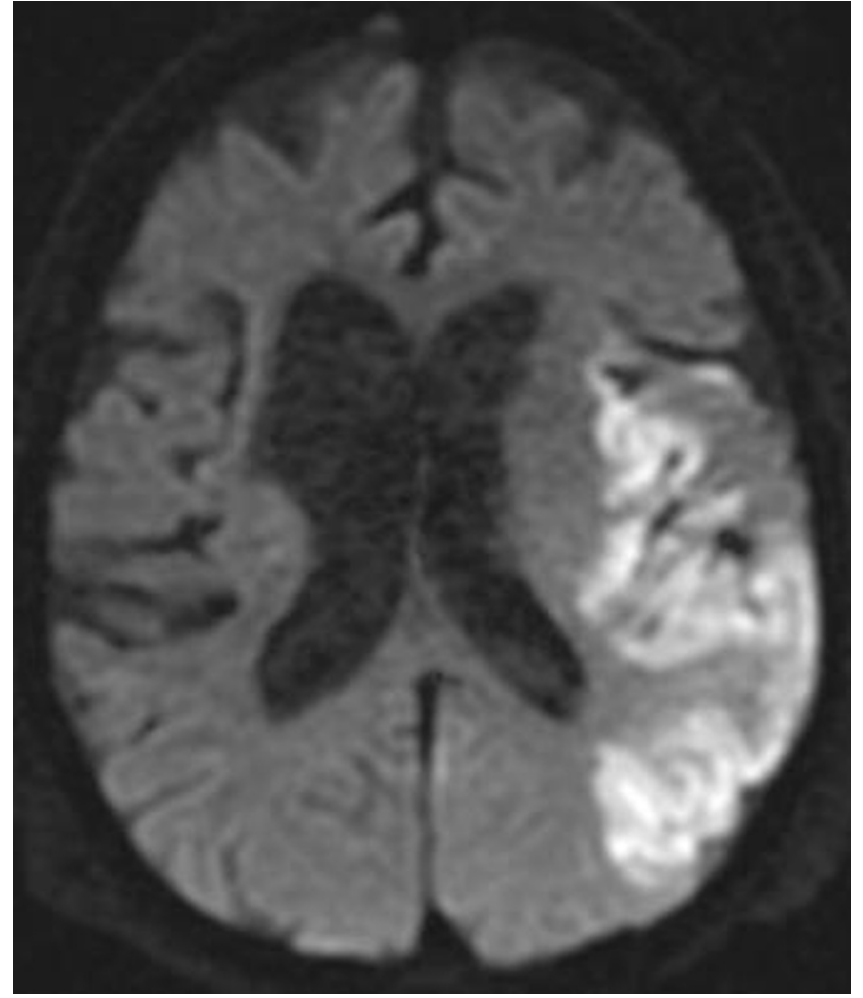

Figure I

Diffusion-weighted image of the brain MRI taken on day 9 with high intensity areas on the left middle cerebral artery region.

treated solely with ciprofloxacin for 8-day period (from day 4 to day 11). Although the patient's neurological deficit ameliorated gradually, she experienced another infarction at the left occipital lobe and the right cerebellar hemisphere on day 18 . Conservative therapy with antibiotics was continued; however, her mental status progressively decreased and blood pressure deteriorated. Blood culture examinations were repeated, but they were all negative. haemodialysis ceased on day 35 due to the unstable haemodynamic status, although treatment with ciprofloxacin and ceftriaxone were continued. On day 39, the patient died of respiratory failure and cardiac arrest. Her clinical course is shown in figure 3. Autopsy revealed a rigid node on the mitral valve (figure 4), associated with septic emboli (mass of gram-negative microorganisms) at the left occipital lobe (figure 5).

\section{Discussion}

Infective endocarditis is a common complication of haemodialysis, the incidence of which is 1.7-2.0 cases/ 1000 patients in France (50-60 times higher than the general population), and 483 cases per 100,000 person-years in the United States of America (74 times higher than the general population) $[3,4]$. The most common causable

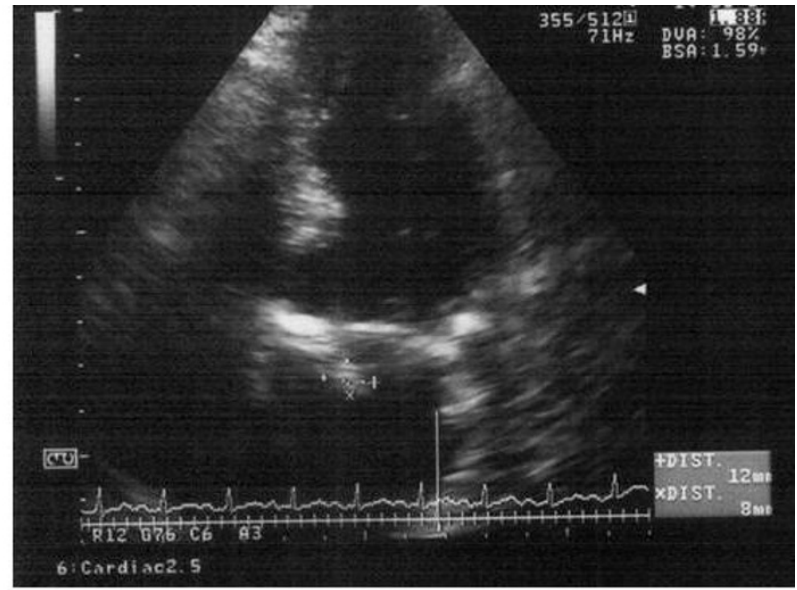

$\mathrm{B}$

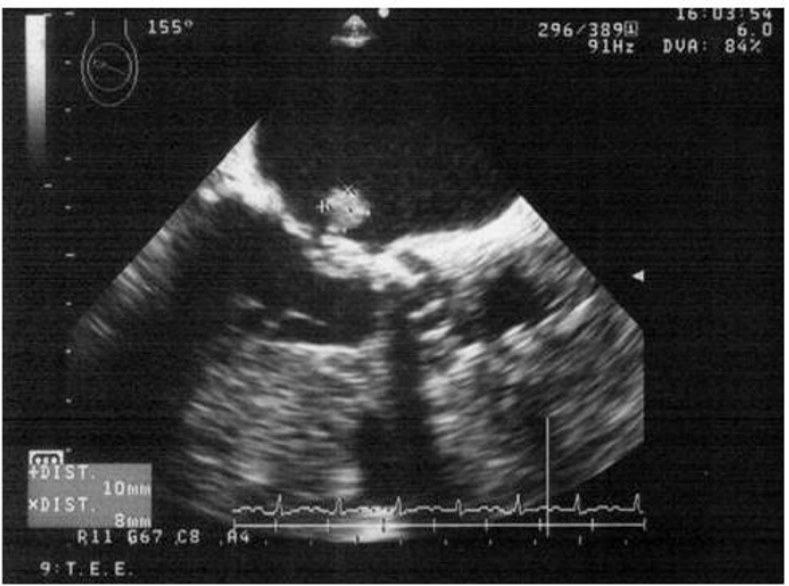

Figure 2

Transthoracic echocardiogram (A) and transesophageal echocardiogram (B) performed on day 13 revealed vegetation on the mitral valve.

pathogen is Staphylococcus aureus, which contributes to 40$80 \%$ of the cases (Table 2). A report from Duke University Medical Center during 1993-1999 demonstrated that the overall proportion of haemodialysis patients out of a total of 329 infective endocarditis patients increased from $6.7 \%$ to $20 \%$ over the 7 year study period. In addition, there was a significant increase in Staphylococcus aureus as the causable agent [5]. We experienced 46 infective endocarditis patients in our hospital during 2003-2008, 4 of which were haemodialysis patients $(8.7 \%)$ [6]. The mitral valve (up to $50 \%$ of cases) and the aortic valve (up to $40 \%$ of cases) are the most commonly affected valves for infective endocarditis in haemodialysis patients [7-9]. To the best of our knowledge, this is the first English report of Salmonella infective endocarditis in a haemodialysis patient.

Gastroenteritis due to Salmonella species occurs relatively frequently; however, it is a rare cause of infective endocar- 


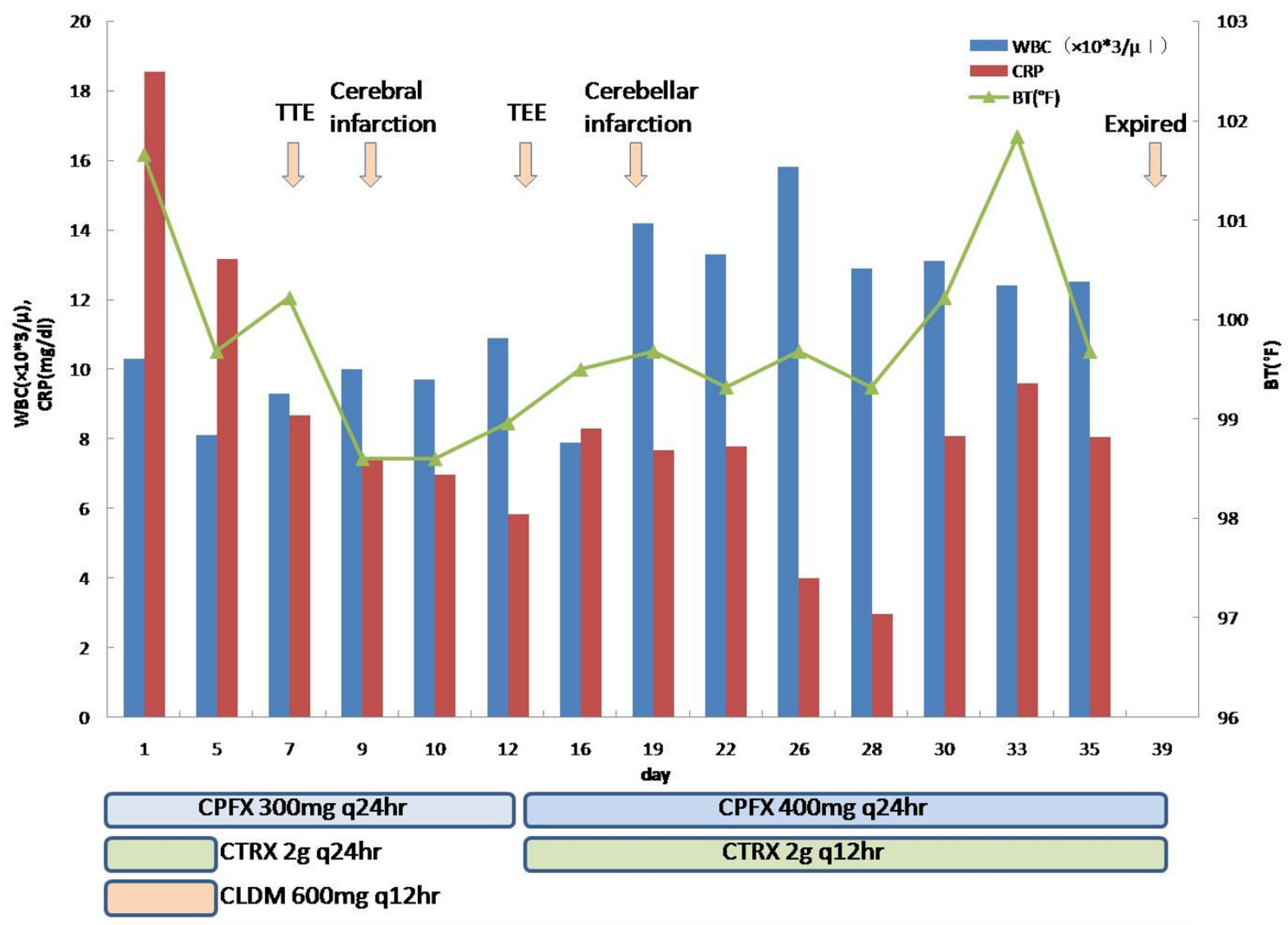

Haemodialysis

Figure 3

Laboratory data, body temperature, and antibiotics administered to the patient after admission to the hospital. Abbreviations: WBC; white blood cell, BT; body temperature, CRP; C-reactive protein, TTE; transthoracic echocardiography, TEE; transesophageal echocardiography, CPFX; ciprofloxacin, CTRX; ceftriaxone, CLDM; clindamycin

ditis. Guerrero identified a total of 72 cases of Salmonella endocarditis within the English literature in 2004; 42 cases before 1987 and 30 cases in 1987-2004 [10]. In a population-based study, Nielsen reported 111 cases of Salmonella bacteremia from 1994 through 2003 in North Jutland Country, Denmark. Among them, there were only 2 cases $(1.8 \%)$ of endocarditis documented [11]. Transient bacteremia is reported to be present in $5-10 \%$ of patients with Salmonella gastroenteritis. Within these cases, the incidence of infective endocarditis is approximately $2.0 \%$ [11].

The intravascular intima is vulnerable to Salmonella species, thus reflecting the ability of salmonella to cause endothelial infections in the presence of atherosclerosis. The risk of endovascular focal infection in elderly patients (age $>50$ ) with Salmonella bacteremia is reported to be 7 -

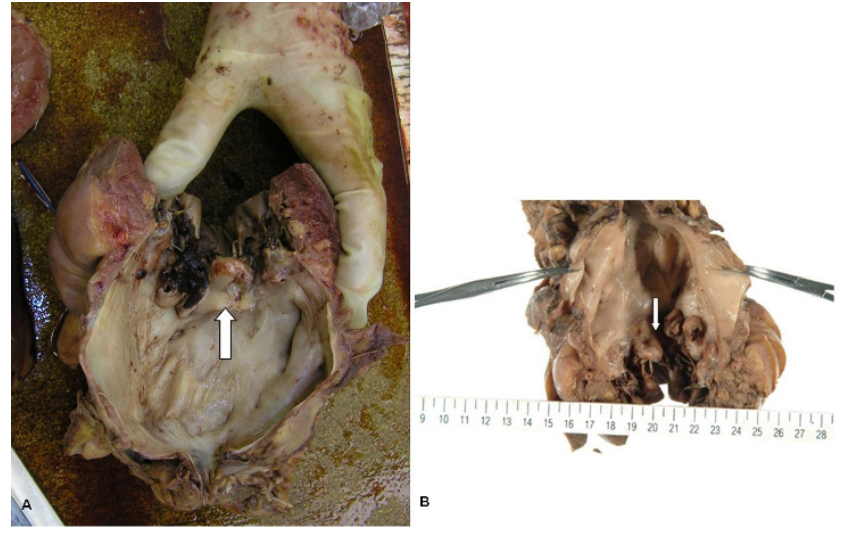

Figure 4

A rigid node on the mitral valve (arrow), found during autopsy $(A, B)$. 


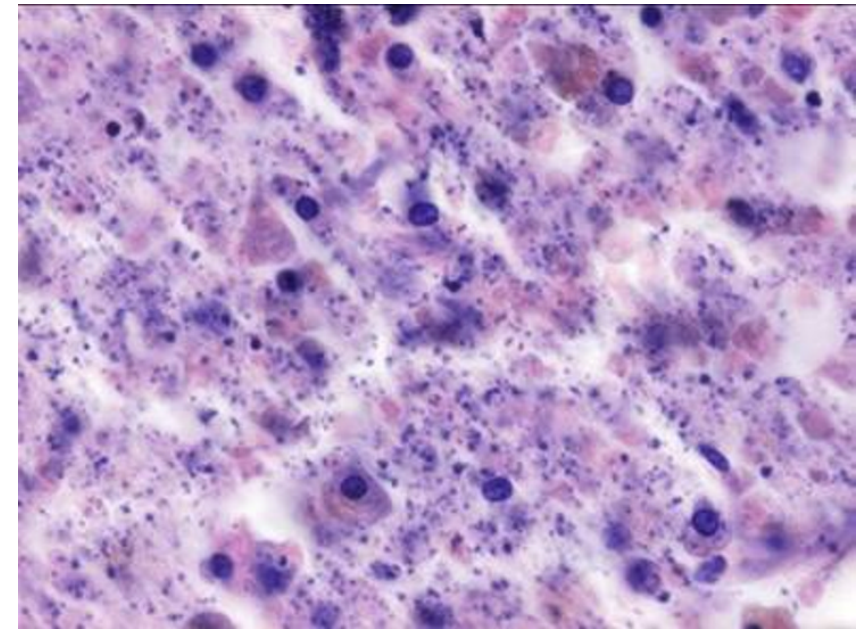

Figure 5

Bacterial proliferation in the cerebral infarction.

$23 \%$ [10-12]. The prognosis of Salmonella endocarditis is improving; Cohen reported the mortality of $69 \%$ (29/42) in 1987 [13], where as Guerrero identified the mortality of $20 \%(6 / 30)$ in 1987-2004 [10]. Guerrero concluded that surgery has played an essential role in reducing the mortality of Salmonella endocarditis, and surgical intervention, including valve replacement, increases survival in Salmonella endocarditis and is the treatment of choice for patients with cardiac failure, persisting sepsis and for those who relapse after discontinuation of antimicrobial therapy. Early surgical intervention may prevent lifethreatening complications including cerebral infarction $[14,15]$, as was observed in the current case. In this case, Salmonella species were suspected from the medical history. Therefore, we began the appropriate treatment of ceftriaxone combined with ciprofloxacin, and performed an early investigation with TTE; however, we failed to detect infective endocarditis before it induced cerebral inf- arction. The cerebral infarction contraindicated her from early surgical intervention. Owing to the increased sensitivity of TEE over TTE in detecting vegetations (Sensitivity for native valve: TTE 60-65\%, TEE 85-95\%) [16], TEE should be performed in any haemodialysis patient with high clinical suspicion of infective endocarditis, and whenever bacteremia with organisms typical for infective endocarditis was detected. It should also be emphasized that in patients with prosthetic valve, TEE is mandatory for those suspected to have infective endocarditis, owing to unacceptably low sensitivity of TTE for detecting infective endocarditis of the prosthetic valve (Sensitivity for prosthetic valve: TTE $<50 \%$, TEE $82-90 \%$ ) [16]. Identification of the optimal treatment for Salmonella endocarditis requires further investigation. In addition, the emergence of ceftriaxone-resistant Salmonella species has recently been reported $[17,18]$. Due to this resistance, combination treatment with a third generation cephalosporin and a fluoroquinolone is offered in life-threatening infections. We treated the patient in this case study with ciprofloxacin, and later combined with ceftriaxone; however, we failed to prevent the patient from developing cerebral infarction, which was identified as septic emboli upon autopsy.

\section{Conclusion}

Salmonella endocarditis has been previously reported in the general population as a rare complication of Salmonella gastroenteritis. To the best of our knowledge, this is the first case-report of Salmonella endocarditis in a haemodialysis patient. Salmonella endocarditis may present an aggressive course; therefore, early surgical intervention is essential for some cases to prevent life-threatening complications. Non-surgical management may treat the patient successfully, but further investigation is required to distinguish patients who require an expeditious surgical intervention from those who can be treated with antibiotics alone.

Table 2: Previously published reports of causable organisms for infective endocarditis in haemodialysis patients

\begin{tabular}{|c|c|c|c|c|c|}
\hline & $\begin{array}{l}\text { Nori et al. } \\
{[17]}\end{array}$ & $\begin{array}{c}\text { Spies et al. } \\
{[9]}\end{array}$ & $\begin{array}{c}\text { Doulton et al. } \\
{[18]}\end{array}$ & $\begin{array}{c}\text { Maraj et al. } \\
{[8]}\end{array}$ & $\begin{array}{c}\text { McCarthy \& Steckelberg } \\
{[7]}\end{array}$ \\
\hline Episodes of infective endocarditis & $N=54$ & $N=40$ & $N=30$ & $N=30$ & $N=17$ \\
\hline Staphylococcus aureus & $40 \%$ & $50 \%$ & $63 \%$ & $80 \%$ & $40 \%$ \\
\hline MRSA & $20 \%$ & $15 \%$ & $7 \%$ & $23 \%$ & \\
\hline CNS & $22 \%$ & $12 \%$ & $13 \%$ & $3 \%$ & $10 \%$ \\
\hline Enterococcus sp. & $33 \%$ & $23 \%$ & $10 \%$ & $7 \%$ & $20 \%$ \\
\hline Streptococcus sp. & & & $10 \%$ & $3 \%$ & $25 \%$ \\
\hline Gram-negative sp. & $13 \%$ & $10 \%$ & & & \\
\hline Candida sp. & & $3 \%$ & & $3 \%$ & \\
\hline Aspergillus sp. & & & & & $5 \%$ \\
\hline Negative blood culture & $2 \%$ & $10 \%$ & & & \\
\hline
\end{tabular}




\section{Competing interests}

The authors declare that they have no competing interests.

\section{Authors' contributions}

YT, MF, KF and YK designed the concepts and design. FT, $\mathrm{YN}$, KT and YK collected the data and contributed to the critical revision of the paper. All authors read and approved the final manuscript.

\section{Acknowledgements}

We thank Uehara $\mathrm{Y}$, Toyama $\mathrm{K}$ and Tamaki $\mathrm{H}$ for their diligent patient care. We also thank Suzuki K (pathological department) for providing us the pictures. Written consent was obtained from the patient's relative for the publication of the study.

\section{References}

I. Cherubin CE, Neu HC, Imperato PJ, Harvey RP, Bellen N: Septicemia with non-typhoid salmonella. Medicine (Baltimore) 1974, 53:365-376.

2. Hohmann EL: Nontyphoidal salmonellosis. Clin Infect Dis 200I, 32:263-269.

3. Hoen B, Alla F, Selton-suty C, et al.: Changing profile of infective endocarditis - results of a one-year survey in France in 1999. JAMA 2002, 288(I):75-8I.

4. Abbott KC, Agodoa LY: Hospitalizations for bacterial endocarditis after initiation of chronic dialysis in the United States. Nephron 2002, 91:203-209.

5. Cabell CH, Jollis JG, Peterson GE, Corey GR, Anderson DJ, Sexton DJ, Woods CW, Reller LB, Ryan T, Fowler VG Jr: Changing patient characteristics and the effect on mortality in endocarditis. Arch Intern Med 2002, 162:90-94.

6. Nishizaki Y, Tsugawa Y, Futatsuyama M, Taki F, Kaneshiro Y, Komatsu Y: Infective Endocarditis in Chronic Kidney Disease Patients. Presentation at 12th Kidney and Cardiovascular Seminar Tokyo, Japan 2008.

7. McCarthy JT, Steckelberg JM: Infective endocarditis in patients receiving long-term hemodialysis. Mayo Clin Proc 2000, 75:1008-1014.

8. Maraj S, Jacobs LE, Kung SC, Raja R, Krishnasamy P, Maraj R, Braitman LE, Kotler MN: Epidemiology and outcome of infective endocarditis in haemodialysis paritents. Am J Med Sci 2002 324:254-260.

9. Spies C, Madison JR, Schatz IJ: Infective endocarditis in patients with end-stage renal disease: clinical presentation and outcome. Arch Intern Med 2004, 164:71-75.

10. Guerrero MLF, Aguado JM, Arribas A, Lumbreras C, Gorgolas M: The spectrum of cardiovascular infections due to Salmonella enterica. A review of clinical features and factors determining outcome. Medicine 2004, 83:123-138.

II. Neilsen H, Gardel KO, Schonheyder HC: High incidence of intravascular focus in nontyphoid Salmonella baccteremia in the age group above $\mathbf{5 0}$ years: a population-based study. APMIS 2006, I | 4(9):64|-645.

12. Soravia-Dunand VA, Loo VG, Salit IE: Aortitis due to Salmonella: report of 10 cases and comprehensive review of the literature. Clin Infect Dis 1999, 29:862-868.

13. Cohen JL, Bartlett JA, Corey GR: Extra-intestinal manifestations of salmonella infections. Medicine (Baltimore) 1987, 66:349-388.

14. Nori US, Manoharan A, Thornby JI, Yee J, Parasuraman R, Ramanathan $\mathrm{V}$ : Mortality risk factors in chronic haemodialysis patients with infective endocarditis. Nephrol Dial Transplant 2006, 2 I:2 184-2190.

I5. Doulton T, Sabharwal N, Cairns HS, Schelenz S, Eykyn S, O'Donnell $P$, Chambers J, Austen C, Goldsmith DJ: Infective endocarditis in dialysis patients: new challenges and old. Kidney Int 2003, 64:720-727.

16. Lester SJ, Wilansky S: Endocarditis and associated complications. Crit Care Med 2007, 35(8 Suppl):S384-39I.

17. Vahaboglu H, Hall LM, Mulazimoglu L, Dodanli S, Yildirim L, Livermore DM: Resistance to extended-spectrum cephalosporins caused by PER-I $\beta$-lactamase, in Salmonella thphimurium from Istanbul, Turkey. J Med Microbiol I 995, 43:294-299.

18. Fey PD, Safranek T], Rupp ME, Dunne EF, Ribot E, Iwen PC, Bradford PA, Angulo FJ, Hinrichs SH: Ceftriaxone-resistant Salmonella infection acquired by a child from cattle. N Engl J Med 2000, 342: $1242-1249$

\section{Pre-publication history}

The pre-publication history for this paper can be accessed here:

http://www.biomedcentral.com/1471-2334/9/161/pre pub
Publish with Biomed Central and every scientist can read your work free of charge

"BioMed Central will be the most significant development for disseminating the results of biomedical research in our lifetime. "

Sir Paul Nurse, Cancer Research UK

Your research papers will be:

- available free of charge to the entire biomedical community

- peer reviewed and published immediately upon acceptance

- cited in PubMed and archived on PubMed Central

- yours - you keep the copyright 